\title{
POWER SERIES HAVING PARTIAL SUMS WITH ZEROS IN A HALF-PLANE ${ }^{1}$
}

\section{ALBERT EDREI}

Introduction. In this note we consider a problem connected with some important work of Pólya [4]. We prove the following

Theorem. Let

$$
a_{0}+a_{1} z+a_{2} z^{2}+\cdots \quad\left(a_{0}=1\right),
$$

be a formal power series which does not reduce to a polynomial.

(i) Assume that, for every positive integer $n$, the zeros of

$$
S_{n}(z)=a_{0}+a_{1} z+a_{2} z^{2}+\cdots+a_{n} z^{n}
$$

lie in a closed half-plane, which is allowed to vary with $n$, but contains the origin on its boundary.

Then

$$
\left|a_{n-1}\right|+\left|a_{n}\right|>0 \quad(n=1,2,3, \cdots)
$$

and

$$
\limsup _{n \rightarrow \infty}\left|a_{n}\right|^{1 / n^{2}}<1 .
$$

It might be of interest to compare this result with the following one, more general but less precise.

Assume that, in the above Theorem, the condition (i) is replaced by the condition

(ii) for every $n(\geqq 1)$, the zeros of $S_{n}(z)$ lie in the angle $\Delta_{n}$ defined by

$$
\theta_{n}-\alpha / 2 \leqq \arg z \leqq \theta_{n}+\alpha / 2 \quad(0 \leqq \alpha<2 \pi) .
$$

Then the function represented by (1) is entire and of order zero:

$$
\lim _{n \rightarrow \infty}\left|a_{n}\right|^{1 / n \log n}=0 .
$$

The latter result is contained in more general ones due to Carlson ${ }^{2}$ [1], Rosenbloom [6; 7], and Korevaar [3].

Received by the editors September 1, 1956 and, in revised form August 15, 1957.

1 This research was supported in part by a grant from the National Science Foundation.

${ }^{2}$ Carlson stated his results in 1924, but published no proofs until 1948 [1]. The first published proofs are due to Weisner [9] in the special case $\alpha<\pi$, and to Rosenbloom [6] in the general case. 
If $\alpha \leqq \pi$, our result ${ }^{3}$ is not only significantly sharper than (5), it is also "best possible."

This may be seen considering the function

$$
\sum_{n=0}^{\infty} a^{-n^{2}} z^{n}
$$

As shown by Pólya and Szegö [3; p. 69, Problem 176], the zeros of this function are all real and negative, so that, even if $\alpha=0$, (4) cannot be improved by replacing $n^{2}$ by a function of more rapid growth.

It would be interesting to know the best possible result in the case $\pi<\alpha<2 \pi$.

1. Inequalities between the coefficients. Let $a_{n} \neq 0$ and consider the polynomial

$$
P_{n}(z)=z^{n}+a_{1}\left|a_{n}\right|^{-1 / n} z^{n-1}+a_{2}\left|a_{n}\right|^{-2 / n} z^{n-2}+\cdots
$$

which by assumption, has all its roots in some half-plane bounded by a straight line through the origin. A lemma of Szász [8; Lemma 3, p. 378] enables us to conclude

$$
\frac{\left|a_{n-k}\right|}{\left|a_{n}\right|^{(n-k) / n}} r^{k} \leqq \max _{|z|=r}\left|P_{n}(z)\right|
$$

$$
\begin{aligned}
& \leqq \exp \left\{r\left|a_{n-1}\right|\left|a_{n}\right|^{-(n-1) / n}\right. \\
& \left.\quad+3 r^{2}\left(\left|a_{n-1}\right|^{2}\left|a_{n}\right|^{-2(n-1) / n}+\left|a_{n-2}\right|\left|a_{n}\right|^{-(n-2) / n}\right)\right\} .
\end{aligned}
$$

Taking $k=n$ and $r=2$, it is clear that (1.1) and the assumptions $a_{0}=1, a_{n} \neq 0$ imply

$$
\left|a_{n-1}\right|+\left|a_{n-2}\right|>0 \text {. }
$$

As (1) is not a polynomial, (1.2) proves (3). In order to prove (4), we put

$$
\left|a_{n}\right|=e^{-\phi(n)} \quad\left(\phi(n)=+\infty \text { if } a_{n}=0\right) .
$$

If $a_{n} \neq 0$, we also introduce

$$
I_{n}=\left|a_{n-1}\right|^{2}\left|a_{n}\right|^{-2(n-1) / n}+\left|a_{n-2}\right|\left|a_{n}\right|^{-(n-2) / n} .
$$

We set, in (1.1),

$$
r=k^{1 / 2} I_{n}^{-1 / 2}
$$

${ }^{3}$ Korevaar [2, pp. 30-31] also investigated the case $\alpha \leqq \pi$; he proved (3) and gave an estimate sharper than (5). 
This is possible because (1.2) implies $I_{n}>0$. We find

$$
\begin{aligned}
& \frac{\left|a_{n-k}\right|}{\left|a_{n}\right|^{(n-k) / n}} k^{k / 2} I_{n}^{-k / 2} \leqq \exp \left(\frac{k^{1 / 2}}{I_{n}^{1 / 2}} \frac{\left|a_{n-1}\right|}{\left|a_{n}\right|^{(n-1) / n}}+3 k\right) \leqq e^{4 / k} \\
& \left|a_{n-k}\right|^{1 / k}\left|a_{n}\right|^{(k-n) / k n} k^{1 / 2} \\
& \quad \leqq 2^{1 / 2} e^{4} \max \left\{\frac{\left|a_{n-1}\right|}{\left|a_{n}\right|^{(n-1) / n}},\left(\frac{\left|a_{n-2}\right|}{\left|a_{n}\right|^{(n-2) / n}}\right)^{1 / 2}\right\}=2^{1 / 2} e^{4} \lambda_{n} .
\end{aligned}
$$

Let $j_{n}$ be the smallest positive integer such that

$$
\lambda_{n}=\left\{\frac{\left|a_{n-j_{n}}\right|}{\left|a_{n}\right|^{\left(n-j_{n}\right) / n}}\right\}^{1 / j_{n}} .
$$

Clearly $j_{n}$ is either 1 or 2 and in view of (1.3), the inequality (1.4) may be rewritten as

$$
\begin{array}{r}
\frac{\phi(n)-\phi(n-k)}{k}+\frac{1}{2}(\log k-\log 2-8) \leqq \\
(k=1,2, \cdots, n) .
\end{array}
$$

The latter inequalities are to be understood in the following sense: to each positive integer $n$ such that $\phi(n) \neq+\infty$, there corresponds an integer $j_{n}$, equal to 1 or 2 , such that

$$
\phi\left(n-j_{n}\right) \neq+\infty,
$$

and such that the inequalities (1.5) are satisfied.

2. Proof of the theorem. Let $p$ be an integer such that

$$
\frac{1}{2}(\log p-\log 2-8)>1 ;
$$

$p$ remains fixed throughout the proof. We first take, in (1.5),

$$
k=n \geqq p \quad \phi(n) \neq+\infty
$$

and obtain

$$
\frac{\phi(n)}{n}-\frac{\phi\left(n-j_{n}\right)}{n-j_{n}}>\frac{j_{n}}{n-j_{n}} .
$$

Hence, we either have

$$
\frac{\phi(n)}{n}-\frac{\phi(n-1)}{n-1}>\frac{1}{n-1},
$$

or else 


$$
\frac{\phi(n)}{n}-\frac{\phi(n-2)}{n-2}>\frac{2}{n-2}>\frac{1}{n-1}+\frac{1}{n-2},
$$

and therefore

$$
\frac{\phi(n)}{n}-\frac{\phi(p-j)}{p-j}>\sum_{\nu=1}^{n-p+j} \frac{1}{n-\nu}
$$

where $j$ is 1 or 2 and $\phi(p-j) \neq+\infty$.

We next introduce a convenient terminology. We say that the real sequence

$$
\psi\left(n_{0}\right), \psi\left(n_{0}+1\right), \psi\left(n_{0}+2\right), \cdots
$$

finite or infinite, has the property $(\mathrm{P})$ if it satisfies the three following conditions:

(i) certain elements of (2.4) may have the value $+\infty$; however, no two consecutive terms are equal to $+\infty$;

(ii) $\psi\left(n_{0}\right)>0, \psi\left(n_{0}+1\right)>0$;

(iii) if $n \geqq n_{0}+2$ and $\psi(n) \neq+\infty$, one at least of the two inequalities $\psi(n)-\psi(n-1)>0, \psi(n)-\psi(n-2)>0$ is satisfied.

As a consequence of these definitions, it is clear that the inequalities

$$
n>m+1 \geqq n_{0}+1
$$

imply the truth of one, at least, of the inequalities

$$
\psi(n)>\psi(m), \quad \psi(n)>\psi(m+1) .
$$

Taking $m=n_{0}$, we see that if a sequence has the property $(\mathrm{P})$, its finite terms are necessarily positive.

We now choose an integer $n_{0}$ such that $\{\phi(n)\}_{n=n_{0}}^{+\infty}$ has the property $(\mathrm{P})$. This is possible in view of (1.2), (1.3), (2.2), and (2.3).

We next put $\psi(n)=\phi(n)-\lambda n^{2}$ and, by an obvious continuity argument, choose $\lambda$ such that

$$
0<\lambda<1 / p
$$

and so small that the finite sequence

$$
\psi\left(n_{0}\right), \psi\left(n_{0}+1\right), \psi\left(n_{0}+2\right), \cdots, \psi\left(n_{0}+p\right)
$$

has the property $(\mathrm{P})$.

If $k$ is either $p$ or $p+1,(2.5)$ implies

$$
\lambda\left(k-j_{n}\right)=\lambda\left\{\frac{n^{2}-\left(n-j_{n}\right)^{2}}{j_{n}}-\frac{n^{2}-(n-k)^{2}}{k}\right\} \leqq \lambda p<1 .
$$

Combining (1.5), (2.1) and (2.7), we find 


$$
\frac{\psi(n)-\psi(n-k)}{k}<\frac{\psi(n)-\psi\left(n-j_{n}\right)}{j_{n}},
$$

which is valid for $n \geqq k, k=p, p+1$.

Hence

$$
\frac{\psi\left(n-j_{n}\right)-\psi(n-k)}{k}<\left(\frac{1}{j_{n}}-\frac{1}{k}\right)\left\{\psi(n)-\psi\left(n-j_{n}\right)\right\} .
$$

If $\phi\left(n_{0}+p+1\right) \neq+\infty$, we may take in (2.8)

This yields

$$
n=n_{0}+p+1 \text {. }
$$

$$
\psi(n)-\psi\left(n-j_{n}\right)>0,
$$

because (2.6) having the property $(\mathrm{P})$, one of the two quantities

$$
\left\{\psi\left(n-j_{n}\right)-\psi(n-p)\right\}, \quad\left\{\psi\left(n-j_{n}\right)-\psi(n-p-1)\right\},
$$

is positive. Hence the sequence $\{\psi(n)\}_{n=n_{0}}^{n_{0}+p+1}$ has the property $(\mathrm{P})$. This enables us to use (2.8) with

$$
n=n_{0}+p+2 \text {, }
$$

and to conclude that $\{\psi(n)\}_{n=n_{0}}^{n_{0}+p+2}$ has the property (P). An obvious induction shows that $\{\psi(n)\}_{n=n_{0}}^{+\infty}$ has the property (P). As this implies the positivity of $\psi(n)$, our theorem is proved.

\section{REFERENCES}

1. F. Carlson, Sur les fonctions entières, Arkiv för Mathematik vol. 35 (1948) pp. $1-18$.

2. J. Korevaar, Approximation and interpolation applied to entire functions, Leiden thesis, Amsterdam, 1949.

3. - The zeros of approximating polynomials and the canonical representation of an entire function, Duke Math. J. vol. 18 (1951) pp. 573-592.

4. G. Polya, Über Annäherung durch Polynome mit lauter reellen Wurzeln, Rend. Circ. Mat. Palermo vol. 36 (1913) pp. 279-295.

5. G. Pólya and G. Szegö, Aufgaben und Lehrsätze aus der Analysis, vol. 2, Berlin, 1925.

6. P. Rosenbloom, Sequences of polynomials, especially sections of power series, Thesis, Stanford University, 1943.

7. - Distribution of zeros of polynomials, Lectures on Functions of a Complex Variable, Ann Arbor, 1955, pp. 265-285.

8. O. Szász, On sequences of polynomials and the distribution of their zeros, Bull. Amer. Math. Soc. vol. 49 (1943) pp. 377-383.

9. L. Weisner, Power series the roots of whose partial sums lie in a sector, Bull. Amer. Math. Soc. vol. 47 (1941) pp. 160-163.

SyRACUSE UNIVERSITY 